Defining and classifying medical error

\section{Defining and classifying medical error: lessons for learning}

K M Sutcliffe

\section{The capacity for learning is directly affected by how potentially dangerous events are interpreted and categorized}

$\mathrm{T}$ he categories used by organizations to classify and sort events are not trivial; they channel attention, shape interpretations, and serve as springboards for action. One example is the way in which organizations categorize small failures. Some organizations classify mistakes that have been caught and corrected with no untoward consequences - such as a near collision in aviation-as a "near miss", a kind of failure that reveals how close the organization came to disaster. Other organizations do just the opposite. They look at a near miss and label it a "close call", seeing it as evidence of success and the ability to avoid disaster. ${ }^{12}$ The problem with this is that organizations that see mishaps as close calls often fail to treat these events as possible warnings that signal areas of vulnerability. By labeling a near miss as a "close call", the cycle of learning is curtailed: beliefs that current operations are adequate to contain disaster are reinforced which, in turn, limits the search for information and also circumscribes actions to safeguard future operations. ${ }^{2}$ Alternatively, when close calls are seen as near misses, it is clearer that the event is evidence of danger in the guise of safety rather than evidence of safety in the guise of danger. ${ }^{12}$

The main message is that learning from experience is harder than it looks, both for individuals and for organizations. The capacity for learning and the accumulation of knowledge is directly affected by how potentially dangerous events are interpreted and categorized, a key point reflected in the paper by Tamuz $e^{~} \mathrm{al}^{3}$ in this issue of QSHC which studied medication errors and how they were reported and handled, particularly by pharmacists. The study reveals a number of intricacies and unintended consequences of error reporting systems in complex healthcare organizations, and shows how the classification of a medication error set into motion a number of organizational routines for gathering and keeping safety related information, for analyzing and investi- gating errors, for rewarding or penalizing personnel, and perhaps, most importantly, for learning from failure events. The authors take seriously the idea that understanding the systemic aspects of error and its prevention requires a methodology that takes into account the understanding of the event by the participants. Thus, they privilege the perspectives of those who provide care. This is a refreshing departure from most studies of error and its incidence which use data from medical records or other archival sources.

Tamuz et al found that pharmacy staff did not consider an error (such as a prescribing error) to be a reportable incident if it was caught and corrected in the pharmacy; medication errors that slipped through the pharmacy and reached the floor or the patient (such as an error of dispensing or administration) were reported to the hospital incident reporting system, were formally investigated, and sometimes resulted in negative consequences for the pharmacist deemed accountable for the mistake. Errors detected and corrected by pharmacy staff were labeled as "interventions" and were considered to be "non-events" - acceptable mistakes that occurred as a natural part of the work flow. "Defining away" potential close calls led to a systematic and severe underestimation of the number of reported medication errors. Intervention data were consolidated, analyzed, and used by pharmacy managers to reward pharmacists who intervened and were also used as the basis for pharmacist education programs and for making improvements to pharmacy practices. However, these data were not shared with other groups such as physicians or others who might benefit from such information, nor were they requested by physicians and others who might be interested in building safer pathways of care. And herein lies a set of important issues that were not (and have not been) addressed in patient safety research. Why didn't pharmacists (and to encourage future interventions), tell what they knew? Why didn't physicians ask pharmacists what they knew? And why didn't physicians ask why they weren't told?

The tendency in organizations is to interpret no news as good news. If one hears nothing one assumes that things are safe, that things are going well. But things may not be safe. Studies of high risk but highly reliable organizations such as nuclear powered aircraft carriers and chemical manufacturers, ${ }^{45}$ which share with medicine similar contextual characteristics and a potential for adverse events that materialize from small failures, suggest that safe operations require a sensitivity to the way in which activities are interrelated. ${ }^{45}$ It is in those relationships, those handoffs where errors accumulate or are caught. Medical professionals are to be praised for their tendency to attack the problem of medical errors by working to improve technical skills, but the locus of the problem lies upstream and downstream from the skilled individual-in the organizing and connecting of activities. $^{45}$

Establishing error incidence rates will be difficult-if not impossible-to achieve given the ambiguities in defining what constitutes medical error and other difficulties with reporting systems shown in the study by Tamuz et al. Future studies may therefore want to focus on understanding strategies to recover from error and ways to create resilient systems that can learn from near misses and mistakes. ${ }^{6}$ Medicine is often driven by the idea that perfection is the ultimate goal and that mistakes are a personal and professional failure. This mindset, while praiseworthy, can blind people to the idea that mistakes are normal and can provide opportunities to learn. However, learning cannot take place in a context where information about mistakes is disconnected, feedback is limited, and where people do not recognize vital interdependencies.

Qual Saf Health Care 2004;13:8-9.

doi: 10.1136/qshc.2003.008987

Correspondence to: K M Sutcliffe, Associate Professor of Organisational Behaviour and Human Resource Management, University of Michigan Business School, Ann Arbor, MI 48109-1234, USA; ksutclif@umich.edu

\section{REFERENCES}

1 March JG, Sproull LS, Tamuz M. Learning from samples of one or fewer. Organ Sci 1991;2:1-13.

2 Weick KE, Sutcliffe KM. Managing the unexpected: assuring high performance in an age of complexity. San Francisco, CA: Jossey-Bass, 2001.

3 Tamuz M, Thomas E, Franchois KE. Defining and classifying medical error: lessons for patient safety reporting systems. Qual Saf Health Care 2004:13:13-20.

4 Weick KE. The reduction of medical errors through mindful interdependence. In: 
Rosenthal MM, Sutcliffe KM, eds. Medical error. What do we know? What do we do? San Francisco, CA: Jossey-Bass, 2002:177-99.
5 Weick KE, Sutcliffe KM, Obstfeld D. Organizing for high reliability: processes of collective

mindfulness. Res Organ Behav 1999;21:81-123.
6 Carthey J, de Leval M, Reason J. Institutional resilience in healthcare systems. Qual Health Care $2001 ; 10: 29-34$

Does appropriate prescribing result in safer care?

\section{Does appropriate prescribing result in safer care?}

\section{Vahé A Kazandjian}

\section{The coming of age of "safety" indicators}

C an safer practices-especially pertaining to medication use-be measured and analyzed within the existing paradigm of performance assessment in health care? More than 20 years of experience in performance measurement and evaluation, primarily through indicators, have built a path upon which the more controversial, challenging, and yet similar concepts of safe practices can indeed proceed. Whether descriptive and qualitative (organizational culture towards punishment when errors are identified ${ }^{1-3}$ ) or quantitative (use of successive measurement methods to build a performance profile $\left.e^{4-6}\right)$, safer practice is affected by the same latent confounders of performance measurement.

The latency of these confounders complicates identification of the explanatory factors regarding the safety of processes. Consequently, and with the recognition that many explanatory factors remain to be uncovered, the evaluation of safety faces at least the following challenges:

- design of the measurement tools;

- methods of analysis;

- identification of linkages between what is done and what happens.

These challenges are also considered by Paton and Lelliott in their paper on the quality of prescribing for psychiatric inpatients in this issue of QSHC. ${ }^{7}$ They present critical first steps in addressing medication use and safety issues. Based on the findings of this paper, a few additional comments on the above three challenges are appropriate.

\section{DESIGN OF THE MEASUREMENT TOOLS}

While the use of indicators (numerator and denominator based constructs) is well accepted in the measurement of healthcare performance, consensus does not exist on how to design robust measures to quantify safety of care. Although there has been much discussion about why the quantification of medication use or medical decision making in general is different from, say, mortality analysis or readmissions pattern evaluation, the core of the discrepancy is probably more philosophical in nature than methodological. Indeed, for most performance measurement indicators currently in use in health care there is an acceptance of relativity and perhaps even determinismspecifically, relativity encompasses the need for adjustment of a performance rates interpretation to the context and the environment. Determinism pertains to a collective belief that, as long as there are patients and healthcare organizations, certain consequences of performance such as infections, readmissions, mortality, and even dissatisfaction are to be expected. In contrast to both relativity and determinism, today's paradigm of safety seems to recommend an uncompromising pursuit of zero tolerance with little consideration for context or historical beliefs and expectations. It is perhaps not a coincidence that, while risk managers have always dealt with the consequences of errors, it is rare to find rate based indicators in risk management. ${ }^{8}$ What one finds is zero tolerance whereby even one mishap that is translated into a lawsuit or a legal conflict is too many. For the risk manager, comparisons between hospitals of frequency of complaints, lawsuits, and legal fees seem irrelevant. All that matters is for them as an organization to minimize or eradicate errors and their consequences.

\section{METHODS OF ANALYSIS}

Measurement methods are derived from the philosophy, clarified at the outset, of how knowledge gleaned about practices and their impact on patients will be used. If zero tolerance is the only way communities, policy makers, and even direct care providers are willing to look at the issue of safety, our challenge will be furthered since the now "traditional" means of measurement through comparison analysis will be of little value. On the other hand, it may be possible that the initial categorical attitude of zero tolerance may prove to be too stringent and often unrealistic. In that case, the path-no matter how serpentine-prepared by previous research on performance measurement can be passable and even conducive to the accumulation of new knowledge in the area of safer practices and their outcome.

\section{IDENTIFICATION OF LINKAGES BETWEEN WHAT IS DONE AND WHAT HAPPENS}

The most challenging exercise in this framework of understanding safer practices is perhaps that of interpretation. Finding causal relationships between processes and outputs or, in general, what has been done and what has resulted remains probabilistic even when replicated across settings. The web of factors that could have influenced an observed outcome is fundamental to identify and classify by the nature of its influence. Methodologically, understanding the relationship between safer practices and better outcomes immediately brings the unit of analysis to the patient level and the clinical and demographic information necessary to incorporate into the web of factors (often latent) and the consequent correlations and analyses. The paper by Paton and Lelliott ${ }^{7}$ attempts to tackle the issue of "true" indicator development in medication use, especially in psychiatry. The authors recognize the difficulties involved in establishing causal relationships between dispensing medication and appropriateness. As this paper deals with medication dispensing in psychiatry, the analysis of prescribing, dispensing, administering, and monitoring of medications represents a formidable challenge. Paton and Lelliott do not discuss "patient safety" but rather they describe dispensing practices only. How variability in these practices would impact on the patient remains hypothetical and requires a separate research focus and design. For now, it is sufficient to recognize that true indicators of medication use can be designed and that indicators can significantly help in performance measurement and 
evaluation. Furthermore, the authors report their observations from a project bound in time and place. The true test of safety indicators will require an ongoing initiative from a project to a program. ${ }^{9}$ Only then will the impact of indicators, changes in practice, and patient outcomes be better addressed. To paraphrase and adapt one of Buddha's sayings: "Where you are today depends on where you were yesterday. Where you will be tomorrow depends on your goals today." And today we still seem to be searching for our common goals regarding safety in health care.

Qual Saf Health Care 2004;13:9-10.

doi: 10.1136/qshc.2003.008698

Correspondence to: V A Kazandiian, Center for Performance Sciences, 6820 Deerpath Road, Elkridge, MD 21075-6234, USA;

vkazandjian@mhaonline.org
Dr Vahe A Kazandijan is the President of the Center for Performance Sciences, a global outcomes research organization, and Adjunct Professor, the Johns Hopkins University Bloomberg School of Public Health, Baltimore, Maryland. $\mathrm{He}$ is the original architect of and is responsible for the Maryland Quality Indicator Project ${ }^{\circledR}(Q \mid P)$, the continuous performance improvement program used worldwide over the last 19 years by more than 1800 healthcare organizations. In the UK alone, over 125 hospitals from the NHS and the private sector have participated in the international component of the QIP since 1992. Dr Kazandiian is an advisor to the WHO, Europe Office, for the development of indicator projects in European countries.

\section{REFERENCES}

1 Mutter M. One hospital's journey toward reducing medication errors. It Comm J Qual Saf 2003;29:279-88.
2 Bumpus L, al-Assat AF. Using pertormance improvement strategies to reduce and prevent medication errors. 1. J Cardiovasc Manag 2003;14:11-15.

3 Taxis K, Barber N. Causes of intravenous medication errors: an ethnographic study. Qual Saf Health Care 2003; 12:343-7.

4 Papastrat K, Wallace S. Teaching baccalaureate nursing students to prevent medication errors using a problem-based learning approach. $J$ Nurs Educ 2003;42:459-64.

5 Croteau RJ. All outcomes should be disclosed. Jt Comm J Qual Saf 2003;29:556-7.

6 Barron WM, Kuczewski MG. Unanticipated harm to patients: deciding when to disclose outcomes. Jt Comm J Qual Saf 2003;29:551-5.

7 Paton C, Lelliott P. The use of prescribing indicators to measure the quality of care in psychiatric inpatients. Qual Saf Health Care 2004;13:40-5.

8 Kachalia A, Shojania KG, Hofer TP, et al. Does full disclosure of medical errors affect malpractice liability? The jury is still out. Jt Comm J Qual Saf 2003;29:503-11

9 Kazandjian VA. Can the sum of projects end up in a program? The strategies that shape quality of care research. Qual Saf Health Care 2002;11:212-3

\section{Readmission to hospital: a measure of quality or outcome?}

\section{A Clarke}

\section{The value of readmission to hospital as a quality indicator is still debatable}

$\mathrm{R}$ eadmission to hospital has often been considered as a possible measure of quality of hospital care. Although its measurement is not always easy, the concept is beguilingly simple. An information manager in health services once described it like this: "I take my car into the garage; if it needs to go back in a short time then that's obviously because they didn't do a good enough job!" At the individual level, undoubtedly readmission can represent a failure or breakdown in plans of care for a particular patient, or the occurrence of an unexpected adverse outcome-for example, readmission for wound infection or deep venous thrombosis after surgery. However, as might be expected, health care is almost always more complicated than this.

A number of factors unrelated to the quality of hospital care can affect the likelihood of readmission. ${ }^{1}$ Patient factors are important, such as the severity, predictability and chronicity of the underlying condition, or levels of comorbidity or social support. Many hospital factors are known to affect the likelihood of a hospital admission (and therefore the likelihood of re-admission) including the proximity of the hospital, the availability of hospital beds, and the availability of intermediate or "step down facilities". The planning of care pathways can also affect the likelihood of readmission. If the care plan for a particular patient includes an underlying awareness of frequent exacerbations for which hospital care is likely to be necessary, then a readmission may itself represent better quality of care. Patients may be receiving intermittent hospital care for a serious chronic or terminal underlying condition, and a pattern of care that includes frequent hospital admission and as much time as possible at home may be entirely appropriate to their needs. In this case, readmission may actually represent more appropriate care and higher quality care. ${ }^{2}$ On the whole, however, readmission is not investigated in the context of improving the care of an individual patient or as a smaller scale "look back" or audit activity. Its appeal is that it appears to be reasonably easily accessible from routine data sources at the macro level in order to allow for large scale comparisons between different hospitals or health plans.

However, both the definition and the measurement of readmission for comparisons of the quality of care between institutions can be fraught with problems. ${ }^{3}$ The most important issue is to be able to separate planned from unplanned readmissions and to identify the reason for readmission clearly so that planned or unavoidable readmissions are excluded from the comparison. Many healthcare databases do not allow for the tracking of patients from one hospital to another. So, for example, if a patient dissatisfied with the care in the hospital which provided the index admission attends a different hospital or care plan, he/she may not appear as a readmission. The usual timing for a definition of readmission is within 28 days of an index admission, but sometimes readmission within 1 year is also considered. At 1 year it is likely to be very hard to track a causal relationship between two hospital admissions in order to relate the reason for the second admission to the quality of care in the first.

Another problem with using readmissions as a measure of quality of care for large scale comparisons is in identifying the rate of readmission. Both the numerator (the number of readmissions within a given time period) and the denominator (the overall number of people admitted to hospital as an index admission and potentially able to be included in the numerator) need to be defined and measured carefully. If there are high levels of 30 day mortality, for example, then the denominator may 
erroneously include people who have died who should not be included in the calculation of the readmission rate.

Research on the association between the quality of inpatient care and early readmission in 12 Veterans Affairs hospitals has shown, however, that readmissions, if carefully measured, may be useful for comparisons of quality of care. Ashton et al ${ }^{4}$ used a large case control design to investigate 14 day unplanned readmissions in men discharged after an index admission for diabetes $(n=593)$, chronic obstructive lung disease $(\mathrm{n}=1172)$, or heart failure $(\mathrm{n}=748)$. Quality of care during the index stay was assessed by patient case note or chart review using quality criteria for the process of care developed by panels of expert physicians. The authors found that readmission was statistically more likely where quality criteria had not been complied with. They quantified the contribution of "substandard care" to the likelihood of readmission and found that one in seven readmissions in patients with diabetes, one in five in patients with heart failure, and one in 12 in patients with obstructive lung disease were attributable to substandard care. In a meta-analysis the same authors ${ }^{5}$ estimated that the summary odds ratio for readmission at 31 days or less after the index admission in 16 homogeneous comparisons of substandard or normative versus normative or exceptional care was 1.55 (1.25-1.92). They concluded that "early readmission is significantly associated with the process of inpatient care"

This is the context for the paper by Luthi et $a l^{6}$ on the value of readmission in predicting process indicators for patients admitted to hospital with heart failure published in this issue of QSHC. The authors investigated patients who had been readmitted to see the extent to which carefully selected process indicators (such as the use of certain diagnostic tests or prescription of various drugs) can be predicted by readmission. They found that readmission did not predict quality of care for patients with heart failure and suggested that there are limitations to the use of readmission as a quality indicator. Unfortunately, they were unable to exclude planned readmissions from their database of patients admitted after the index admission. However, their findings continue to cast doubt on the value of readmission as a quality indicator.

In the end, the main problem with the use of readmission as a measure of quality is that it is always going to be an unsatisfactory proxy for measuring either quality or outcome. Whether a patient is readmitted or not is surely less important than whether he or she has a satisfactory outcome of the index hospital stay, measured using valid and reliable indicators of health status or quality of life. ${ }^{1}$ The time must come when we give up measuring unsatisfactory performance indicators simply because they are available and, instead, concentrate harder on allowing for known valid measures of the quality of care to be collected as a matter of routine.

Qual Saf Health Care 2004;13:10-11. doi: 10.1136/qshc.2003.008789

Correspondence to: A Clarke, Institute of Community Health Sciences, Queen Mary, Barts and the London School of Medicine and Dentistry, London El 4NS;

a.e.clarke@qmul.ac.uk

\section{REFERENCES}

1 Milne RIG, Clarke A. Can readmission rates be used as an outcome indicator? BMJ 1990;301:1139-40

2 Clarke A. Are readmissions avoidable? BMJ 1990;301:1136-8.

3 Chambers M, Clarke A. Readmissions: measuring rates. BMJ 1990:301:1134-6.

4 Ashton CM, Kuykendall DH, Johnson ML, et al. The association between the quality of inpatient care and early readmission. Ann Intern Med 1995: 122:415-21.

5 Ashton CM, del Junco DJ, Mansyur C, et al. The association between the quality of in-patient care and early readmission: a meta-analysis of the evidence. Med Care 1997;35:1044-59.

6 Luthi JC, Burnand B, McClellan WM, et al. Is readmission to hospital an indicator of poor process of care for patients with heart failure? Qual Saf Health Care 2004;13:46-51.

\section{Preserving moral quality in research, audit, and quality improvement} L Doyal

$M$ ost discussions about the ethics of healthcare research focus on the possible harm that could be done to participants. Sometimes such deliberations will highlight tensions between the clinical duty of care to protect the life and health of individual patients to the highest standard and the need to engage in the research that makes improvements in health care possible. Because of the potential risks, patients should not be involved in research unless they have given their informed consent. Equally, they should not be asked to participate unless the project itself has been passed through a process of independent review and its risks assessed. The moral principles for such reviews are summarised in the Declaration of Helsinki. This states, among other things, that consent is only valid if it is based on detailed and appropriate information, that risks should always be proportional to potential benefit, that confidentiality should be protected, and that the interests of individuals should never be compromised solely in order to further the interests of the public.

The ethical review of healthcare research is carried out by research ethics committees (RECs) designed to implement the Helsinki principles. Few now seriously question the moral importance of this work and its centrality for sustaining the trust of research participants. While there may be criticisms of the effectiveness and efficiency of RECs, these are usually arguments for their improvement rather than against their very existence. Some healthcare professionals will always be uncomfortable with any review process that may question the moral quality of their practice. However, such discomfort is a small price to pay for achieving the more important goal of respecting human rights and reinforcing the willingness of all the relevant parties to participate in research to improve health care.

However, research is not the only activity necessary for achieving medical progress. While this work contributes to the creation of new knowledge and skills, strategies for carrying out audit and quality improvement (AQI) are also essential if these innovations are to be delivered to patients in the most appropriate ways. AQI can take a variety of forms from regular reviews of the clinical results of individual practitioners to ongoing assessments of the successes and failures of particular delivery systems and the development 
of strategies for their improvement. Just as it is unethical to engage in research on humans that cannot achieve its stated goals, it is also immoral to squander the potential of good research through the use of ineffective or hazardous methods to deliver it.

As the well referenced paper in this issue by Joanne Lynne demonstrates, AQI may also pose problems of its own within the terms of the Helsinki principles. ${ }^{1}$ For example, the confidentiality of medical records, patients, or staff may be breached and the wording of questionnaires may distress relatives or staff. Although these and other risks may not be as great as those associated with more conventional forms of medical research, they do need serious consideration. We need to ask whether the same moral principles applied to research proposals should be used to review proposals for AQI. A good deal of confusion surrounds this issue, along with that of how to distinguish between research and AQI. ${ }^{2}$ Certainly, if already over-stretched RECs also had to review AQI projects, they would have even less time for looking at research proposals that could potentially pose more serious hazards. Moreover the time and energy required would involve a risk of stifling new AQI initiatives. ${ }^{3}$ Yet as long as AQI strategies continue to pose at least some of the same ethical problems as research, Lynn rightly argues that a way must be found of striking the right moral balance between respect for the rights of participants and the duty to protect them and anyone else who might be potentially harmed. This will require careful assessment of different types of AQI and their risk potential.

Many proposals for AQI are so ethically benign that full REC evaluation would be absurd. An example would be a limited review of medical records with strict methods in place for the protection of confidentiality and no intent to make any further contact with patients. But even here, patients should be given explicit information about the ongoing process of quality review and why it is in the interests of everyone involved. Through a policy of opt out linked to an effective information campaign, they should have the option not to participate in such a review (although refusals are unlikely to happen if the information provided is clear). It is increasingly accepted that some element of choice should always be present when patients or staff are used to facilitate any form of healthcare inquiry. There are a variety of feasible and practical ways of achieving this as regards AQI.

Where full REC review is deemed inappropriate for an AQI project, some form of ethical review may still be appropriate. Of course, this often occurs informally during the process of project design. For example, most AQI practitioners will take care to minimise the potential distress of their questionnaires and to properly protect the privacy of those who complete them. However, review of this kind should be formalised to ensure the independence of the process. ${ }^{4}$ There is no reason to assume that this would be institutionally burdensome if it was properly organised. Were it to be so, academic journals that refuse to publish AQI results without REC approval would look silly. Until it is, they may have a point.

One very important reason for ensuring the independent review of AQI as well as research is that the line between them can sometimes become blurred. For example, as Lynn observes, although some studies may be designed only to investigate particular examples of healthcare delivery they may still propose controls and the randomisation of the population under investigation. Such proposals should be sent to a REC. ${ }^{5}$
Any form of selective intervention within health care can lead to distress and a breakdown of trust, even when there is little or no chance of other forms of harm. ${ }^{6}$ It is therefore of great importance that high standards of moral assessment-especially those concerning consent and confidentiality-are seen to be applied before studies of this kind are allowed to proceed.

Of course, requiring appropriate ethical review of all AQI initiatives' methods may result in some of this work not taking place. The same point applies to the requirement that all healthcare research is reviewed by RECs. If this means that the rights and dignity of those who receive and deliver health care are protected-and that the moral quality of health care is preserved-it is a price worth paying.

Qual Saf Health Care 2004;13:11-12. doi: 10.1136/qshc. 2003.008680

Correspondence to: Professor L Doyal, Human Science and Medical Ethics, Queen Mary, University of London, London El 2AD, UK; l.doyal@qmul.ac.uk

\section{REFERENCES}

1 Lynn J. When does quality improvement count as research? Human subjects protections and theories of knowledge. Qual Saf Health Care 2004; 13:67-70

2 Wilson A, Baker R, Thompson J. Differentiating between audit and research: postal survey of health authorities' views. BMJ 1999:319:1235.

3 Cretin S, Lynn J, Batalden PB, et al. Should patients in quality improvement activities have the same protections as participants in research studies? JAMA 2000;284:1786.

4 National Health \& Medical Research Council. When does quality assurance in health care require independent ethical review? Canberra: NHMRC, 2003:1-13.

5 Bellin E, Dubler NN. The quality improvementresearch divide and the need for external oversight. Am J Public Health 2001;91:1512-7.

6 Doyal L. Journals should not publish research to which patients have not given fully informed consent-with three exceptions. BM 1997;314:1107-11. 